IJMMS 30:9 (2002) 511-514

PII. S0161171202111252

http://ijmms.hindawi.com

(c) Hindawi Publishing Corp.

\title{
SOME THEOREMS OF RANDOM OPERATOR EQUATIONS
}

\section{ZHU CHUANXI and XU ZONGBEN}

Received 17 January 2002

We investigate a class of random operator equations, generalize a famous theorem, and obtain some new results.

2000 Mathematics Subject Classification: 60H25, 47H10.

Let $E$ be a separable real Banach space, $(\mathbf{E}, \mathbf{B})$ a measurable space, where $\mathbf{B}$ denotes the $\sigma$-algebra generated by all open subsets in $E$, let $(\Omega, U, \gamma)$ be a complete probability measure space, where $\gamma(\Omega)=1$, let $D$ be a bounded open set in $X$ and $\partial D$ the boundary of $D$ in $X$. Let $X$ be a cone in $E$, and let " $\leq$ ", "<" be partial order of $E$.

LEMMA 1. When $y>1, \alpha>0, x \in X$, and $x \neq \theta$, the following inequality holds:

$$
(y-1)^{\alpha+1} x<y^{\alpha+1} x-x .
$$

Proof. Letting $f(y)=y^{\alpha+1}-1-(y-1)^{\alpha+1}$, where $\alpha>0$, then

$$
\begin{aligned}
f^{\prime}(y) & =(\alpha+1) y^{\alpha}-(\alpha+1)(y-1)^{\alpha} \\
& =(\alpha+1)\left[y^{\alpha}-(y-1)^{\alpha}\right]>0
\end{aligned}
$$

(since $y>1$, then $0<y-1<y$, and $\alpha>0$, obtaining $0<(y-1)^{\alpha}<y^{\alpha}$, i.e., $y^{\alpha}-$ $\left.(y-1)^{\alpha}>0\right)$.

Therefore $f(y)$ is a monotonous increasing function. When $y>1$, we have $f(y)>$ $f(1)$, and $f(1)=0$. Hence $f(y)>0$, that is, $y^{\alpha+1}-1-(y-1)^{\alpha+1}>0$, that is,

$$
(y-1)^{\alpha+1}<y^{\alpha+1}-1 .
$$

When $x \in X, x \neq \theta$, that is, $x>\theta$, we have $(y-1)^{\alpha+1} x<y^{\alpha+1} x-x$.

LEMmA 2 (see [1]). Let $X$ be a closed convex subset of $E, D$ a bounded open subset in $X$, and $\theta \in D$. Suppose that $A: \Omega \times \bar{D} \rightarrow X$ is a random semiclosed 1-set-contractive operator. Meanwhile, such that $x \neq(t / \mu) A(\omega, x)$ a.s., for every $\omega \in \Omega$, for every $x \in$ $\partial D$, where $t \in(0,1], \mu \geq 1$. Then the random operator equation $A(\omega, x)=\mu x$, (for every $(\omega, x) \in \Omega \times \bar{D}, \mu \geq 1$ ) has a random solution in $D$.

TheOrem 3. Let $D$ be a bounded open subset in $X$ and $\theta \in D$. Suppose that $A$ : $\Omega \times \bar{D} \rightarrow X$ is a random semiclosed 1-set-contractive operator, such that

$$
\begin{aligned}
{[\lambda\|\mu x\|} & \left.+\|A(\omega, x)-\mu x\|^{\alpha}\right]\|A(\omega, x)-\mu x\| x \\
& \geq\left[\lambda\|\mu x\|+\|A(\omega, x)\|^{\alpha}\right]\|A(\omega, x)\| x-\lambda\|\mu x\|^{2} x-\|\mu x\|^{\alpha+1} x
\end{aligned}
$$


for every $(\omega, x) \in \Omega \times \partial D, \lambda \geq 0, \mu \geq 1, \alpha>0$. Then the random operator equation $A(\omega, x)=\mu x$ (for every $(\omega, x) \in \Omega \times \bar{D}$, where $\mu \geq 1$ ) has a random solution in $\bar{D}$.

Proof. Assume that $A(\omega, x)=\mu x$ has no random solution on $\partial D$ (otherwise, the theorem has obtained proof), that is, $A(\omega, x) \neq \mu x$ a.s., for every $(\omega, x) \in \Omega \times \partial D$, where $\mu \geq 1$. That is,

$$
x \neq \frac{1}{\mu} A(\omega, x) \quad \text { a.s. }
$$

We prove that

$$
x \neq t \frac{1}{\mu} A(\omega, x),
$$

where $\mu \geq 1, t \in(0,1)$, for every $(\omega, x) \in \Omega \times \partial D$.

Suppose that (6) is not true, that is, there exists a $t_{0} \in(0,1)$, an $\omega_{0} \in \Omega$, and an $x_{0} \in \partial D$, such that $x_{0}=t_{0}(1 / \mu) A\left(\omega_{0}, x_{0}\right)$. That is, $A\left(\omega_{0}, x_{0}\right)=\left(\mu / t_{0}\right) x_{0}$, where $\mu \geq 1$, $t_{0} \in(0,1), \omega_{0} \in \Omega$, and $x_{0} \in \partial D$.

Inserting $A\left(\omega_{0}, x_{0}\right)=\left(\mu / t_{0}\right) x_{0}$ into (4), obtaining

$$
\begin{aligned}
& {\left[\lambda\left\|\mu x_{0}\right\|+\left\|\frac{\mu}{t_{0}} x_{0}-\mu x_{0}\right\|^{\alpha}\right]\left\|\frac{\mu}{t_{0}} x_{0}-\mu x_{0}\right\| x_{0}} \\
& \geq\left[\lambda\left\|\mu x_{0}\right\|+\left\|\frac{\mu}{t_{0}} x_{0}\right\|^{\alpha}\right]\left\|\frac{\mu}{t_{0}} x_{0}\right\| x_{0}-\lambda\left\|\mu x_{0}\right\|^{2} x_{0}-\left\|\mu x_{0}\right\|^{\alpha+1} x_{0},
\end{aligned}
$$

where $\lambda \geq 0, \mu \geq 1, \alpha>0, t_{0} \in(0,1)$, and $x_{0} \in \partial D$. This implies that

$$
\begin{aligned}
& \lambda\left\|\mu x_{0}\right\|\left\|\frac{\mu}{t_{0}} x_{0}-\mu x_{0}\right\| x_{0}+\left\|\frac{\mu}{t_{0}} x_{0}-\mu x_{0}\right\|^{\alpha+1} x_{0} \\
& \geq \lambda\left\|\mu x_{0}\right\|\left\|\frac{\mu}{t_{0}} x_{0}\right\| x_{0}+\left\|\frac{\mu}{t_{0}} x_{0}\right\|^{\alpha+1} x_{0}-\lambda\left\|\mu x_{0}\right\|^{2} x_{0}-\left\|\mu x_{0}\right\|^{\alpha+1} x_{0},
\end{aligned}
$$

that is,

$$
\begin{aligned}
& \lambda\left(\frac{1}{t_{0}}-1\right)\left\|\mu x_{0}\right\|^{2} x_{0}+\left(\frac{1}{t_{0}}-1\right)^{\alpha+1}\left\|\mu x_{0}\right\|^{\alpha+1} x_{0} \\
& \quad \geq \lambda\left(\frac{1}{t_{0}}-1\right)\left\|\mu x_{0}\right\|^{2} x_{0}+\frac{1}{t_{0}^{\alpha+1}}\left\|\mu x_{0}\right\|^{\alpha+1} x_{0}-\left\|\mu x_{0}\right\|^{\alpha+1} x_{0}
\end{aligned}
$$

since $\mu \geq 1, x_{0} \in \partial D$, thus $\mu x_{0} \neq 0$.

Therefore $\left\|\mu x_{0}\right\|^{\alpha+1} \neq 0$, by (9), we obtain

$$
\left(\frac{1}{t_{0}}-1\right)^{\alpha+1} x_{0} \geq \frac{1}{t_{0}^{\alpha+1}} x_{0}-x_{0} .
$$


Letting $y=1 / t_{0}$, by (10), we have

$$
(y-1)^{\alpha+1} x_{0} \geq y^{\alpha+1} x_{0}-x_{0}
$$

where $y>1, \alpha>0, x_{0} \in X$, and $x_{0} \neq \theta$.

This is in contradiction with Lemma 1 . Hence

$$
x \neq t \frac{1}{\mu} A(\omega, x)
$$

for every $(\omega, x) \in \Omega \times \partial D$, where $t \in(0,1), \mu \geq 1$.

By (5) and (12), we know that

$$
x \neq t \frac{1}{\mu} A(\omega, x) \quad \text { a.s., }
$$

where $\mu \geq 1, t \in(0,1)$, for every $(\omega, x) \in \Omega \times \partial D$.

According to Lemma 2 , we obtain that the random operator equation $A(\omega, x)=\mu x$ (where $\mu \geq 1$, for every $(\omega, x) \in \Omega \times \bar{D}$ ) has a random solution in $D$.

REMARK 4. In Theorem 3 , when $\lambda=0, \alpha=1, \mu=1$, and $A(\omega, \cdot)=A$, (4) is that $\|A x-x\|^{2} \geq\|A x\|^{2}-\|x\|^{2}$. Thus, Theorem 3 is a generalization of the famous Altman theorem.

We can see that Lemma 5 holds easily.

LEMmA 5. When $y>1, \alpha>0, x \in X$, and $x \neq \theta$, the following inequality holds:

$$
(y+1)^{\alpha+1} x>y^{\alpha+1} x+x .
$$

Theorem 6. Let $D$ be a bounded open subset in $X$ and $\theta \in D$. Suppose that $A$ : $\Omega \times \bar{D} \rightarrow X$ is a random semiclosed 1-set-contractive operator, such that

$$
\begin{aligned}
& {\left[\lambda\|\mu x\|+\|A(\omega, x)+\mu x\|^{\alpha}\right]\|A(\omega, x)+\mu x\| x} \\
& \quad \leq\left[\lambda\|\mu x\|+\|A(\omega, x)\|^{\alpha}\right]\|A(\omega, x)\| x+\lambda\|\mu x\|^{2} x+\|\mu x\|^{\alpha+1} x,
\end{aligned}
$$

where $\lambda \geq 0, \mu \geq 1, \alpha>0$, for every $(\omega, x) \in \Omega \times \partial D$. Then the random operator equation $A(\omega, x)=\mu x$ (where $\mu \geq 1$, for every $(\omega, x) \in \Omega \times \bar{D}$ ) has a random solution in $D$.

Proof. From (15), we can easily prove that $A(\omega, x)=\mu x$ has no random solution on $\partial D$, by virtue of Lemma 5 , see Theorem 3 for other section.

LEMMA 7. When $y>1, \alpha>0, x \in X$, and $x \neq \theta$, the following inequality holds:

$$
(y+1)^{\alpha+1} x-(y-1)^{\alpha+1} x>2 x .
$$

Proof. By Lemmas 1 and 5, we have

$$
\begin{gathered}
(y-1)^{\alpha+1} x<y^{\alpha+1} x-x, \\
y^{\alpha+1} x+x<(y+1)^{\alpha+1} x,
\end{gathered}
$$


summing (17) and (18) we obtain

$$
(y-1)^{\alpha+1} x+y^{\alpha+1} x+x<y^{\alpha+1} x-x+(y+1)^{\alpha+1} x .
$$

That is,

$$
(y+1)^{\alpha+1} x-(y-1)^{\alpha+1} x>2 x,
$$

where $\alpha>0, y>1, x \in X$, and $x \neq \theta$.

TheOrem 8. Let $D$ be a bounded open subset in $X$ and $\theta \in D$. Suppose that $A$ : $\Omega \times \bar{D} \rightarrow X$ is a random semiclosed 1-set-contractive operator, such that

$$
\|A(\omega, x)+\mu x\|^{\alpha+1} x-\|A(\omega, x)-\mu x\|^{\alpha+1} x \leq 2\|\mu x\|^{\alpha+1} x,
$$

where $\alpha>0, \mu \geq 1$, for every $(\omega, x) \in \Omega \times \partial D$. Then the random operator equation $A(\omega, x)=\mu x$ (where $\mu \geq 1$, for every $(\omega, x) \in \Omega \times \bar{D}$ ) has a random solution in $D$.

Proof. The theorem can be proved using Lemma 7, see also Theorems 3 and 6 .

REMARK 9. Since $X$ is a cone in $E$, then $X$ is a closed convex subset of $E$.

ACKNOWLEDGMENT. This work was funded by the Natural Science Foundation of China.

\section{REFERENCES}

[1] C. X. Zhu, Some theorems on random operator equations of 1-set-contractive type, Adv. in Math. (China) 27 (1998), no. 5, 464-468.

ZHu CHUANXI: RESEARCH CENTER FOR APPLIED MATHEMATICS AND INSTITUTE FOR INFORMATION AND SYSTEM SCIENCE, XI'AN JiAOTONG UNIVERSITY, XI'AN 710049, CHINA; INSTITUTE OF MATHEMATICS, NANCHANG UNIVERSITY, NANCHANG 330029, CHINA

XU ZONGBEN: RESEARCH CENTER FOR APPLIED MATHEMATICS AND INSTITUTE FOR INFORMATION AND SYSTEM SCIENCE, XI'AN JIAOTONG UNIVERSITY, XI'AN 710049, CHINA 


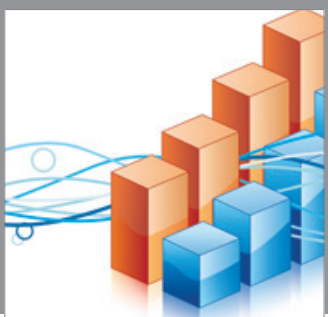

Advances in

Operations Research

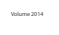

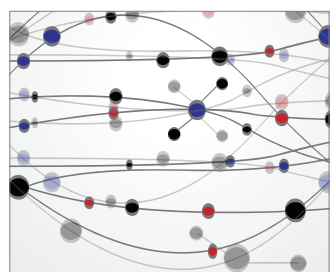

\section{The Scientific} World Journal
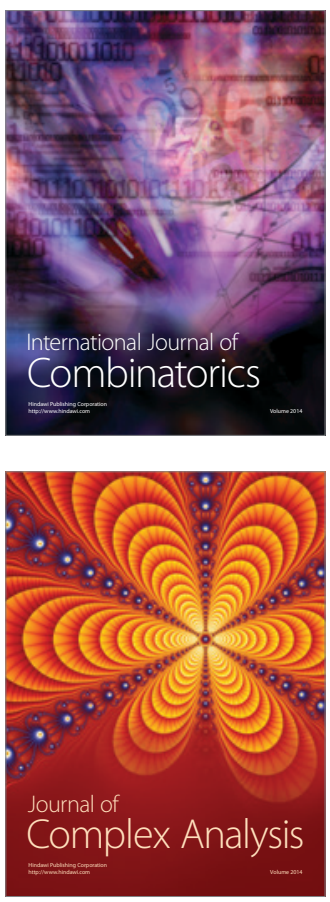

International Journal of

Mathematics and

Mathematical

Sciences
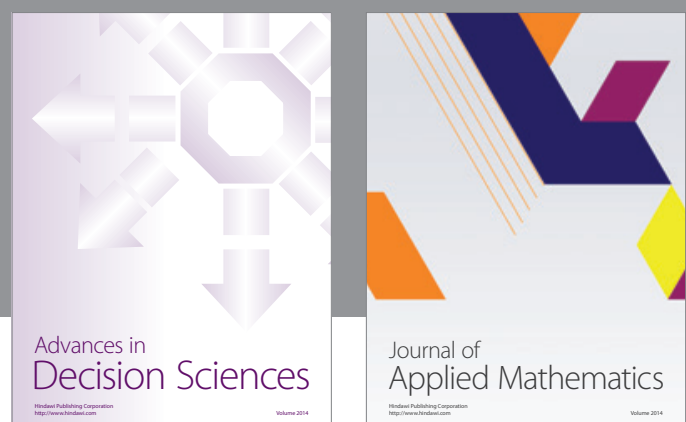

Journal of

Applied Mathematics
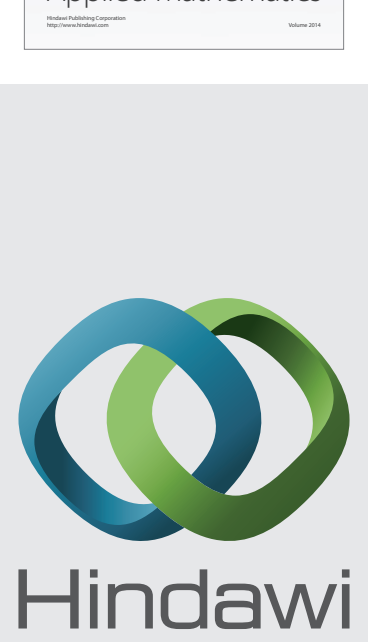

Submit your manuscripts at http://www.hindawi.com
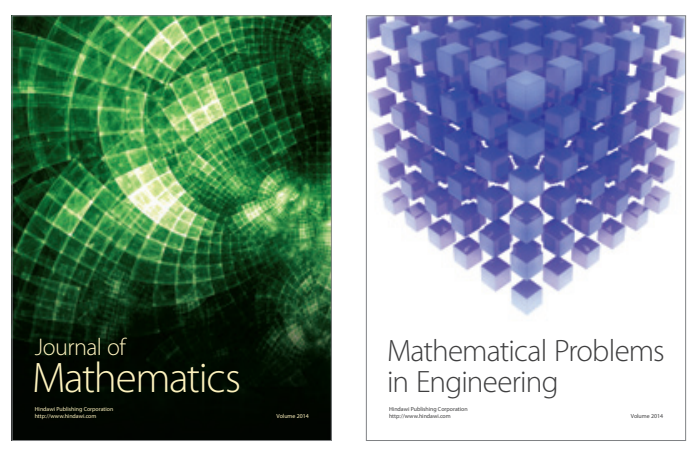

Mathematical Problems in Engineering
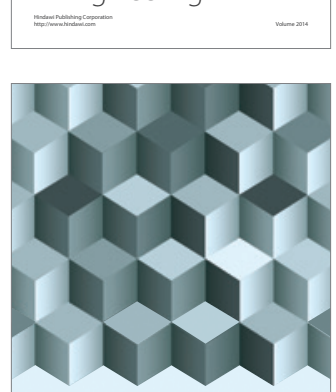

Journal of

Function Spaces
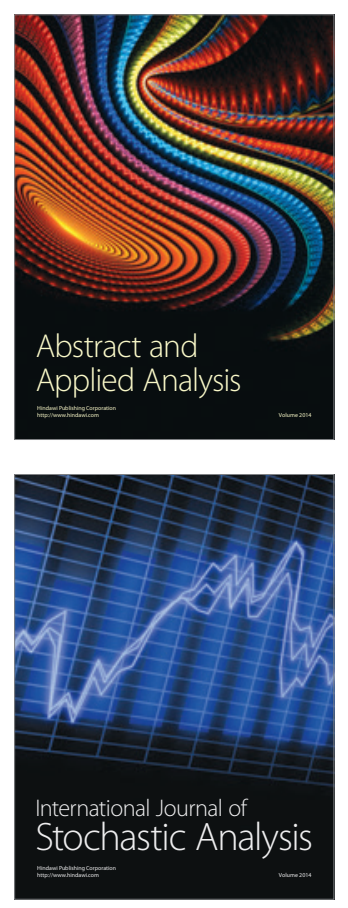

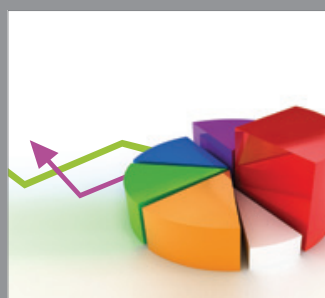

ournal of

Probability and Statistics

Promensencen
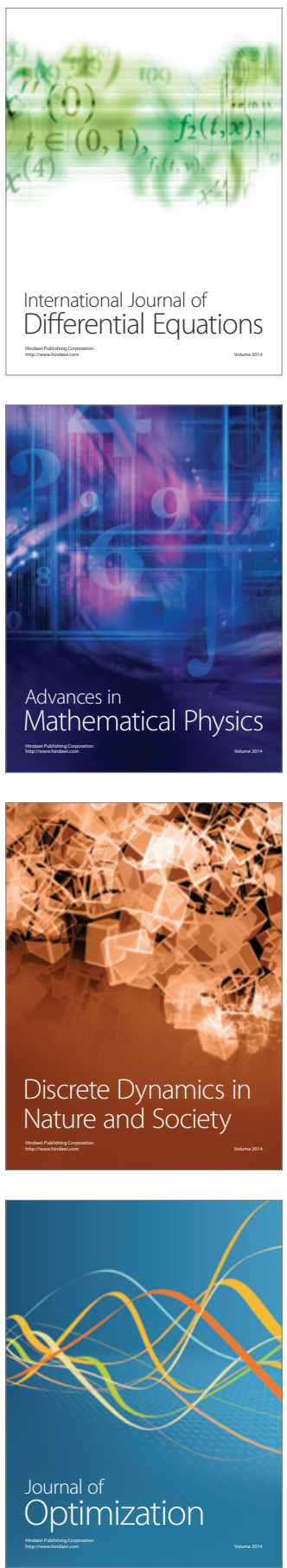